\title{
Deep learning for image-based large-flowered chrysanthemum cultivar recognition
}

\author{
Zhilan Liu', Jue Wang ${ }^{1}$, Ye Tian ${ }^{2^{*}}$ and Silan Dai ${ }^{1^{*}}$ (D)
}

\begin{abstract}
Background: Cultivar recognition is a basic work in flower production, research, and commercial application. Chinese large-flowered chrysanthemum (Chrysanthemum $\times$ morifolium Ramat.) is miraculous because of its high ornamental value and rich cultural deposits. However, the complicated capitulum structure, various floret types and numerous cultivars hinder chrysanthemum cultivar recognition. Here, we explore how deep learning method can be applied to chrysanthemum cultivar recognition.

Results: We propose deep learning models with two networks VGG16 and ResNet50 to recognize large-flowered chrysanthemum. Dataset A comprising 14,000 images for 103 cultivars, and dataset B comprising 197 images from different years were collected. Dataset A was used to train the networks and determine the calibration accuracy (Top-5 rate of above 98\%), and dataset B was used to evaluate the model generalization performance (Top-5 rate of above 78\%). Moreover, gradient-weighted class activation mapping (Grad-CAM) visualization and feature clustering analysis were used to explore how the deep learning model recognizes chrysanthemum cultivars.
\end{abstract}

Conclusion: Deep learning method applied to cultivar recognition is a breakthrough in horticultural science with the advantages of strong recognition performance and high recognition speed. Inflorescence edge areas, disc floret areas, inflorescence colour and inflorescence shape may well be the key factors in model decision-making process, which are also critical in human decision-making.

Keywords: Chrysanthemum × morifolium Ramat., Deep learning, Image recognition, Grad-CAM

\section{Background}

Chrysanthemum $\times$ morifolium Ramat., which originated in China, has high ornamental and commercial value in the floriculture industry around the world [1-3], and its major production areas cover China, Japan, the Netherlands and South Korea [4]. The Chinese large-flowered chrysanthemum cultivar group is one of the largest cultivar groups of chrysanthemum [5]. The growing number of new chrysanthemum cultivars bred around the world has made it harder to recognize, even if professional researchers may confuse chrysanthemum cultivar,

\footnotetext{
*Correspondence: tytoemail@sina.com; silandai@sina.com

${ }^{1}$ College of Landscape Architecture, Beijing Forestry University,

Beijing 100083, China

${ }^{2}$ College of Technology, Beijing Forestry University, Beijing 100083, China
}

which caused severe loopholes in the management and protection of chrysanthemum resources. Many difficulties have been encountered in the cultivar recognition of large-flowered chrysanthemums, due to the large number of cultivars $[2,5]$ (Fig. 1a), the complex capitulum structure, the various floret types [6-8] (Fig. 1b), and the highly heterozygous genetic background $[9,10]$. Thus, it is extremely challenging to recognize chrysanthemums accurately and rapidly.

In previous studies, the traditional morphological method, the comprehensive mathematical statistics method and molecular markers were used to solve the large-flowered chrysanthemum recognition problem. The traditional method utilized human decisionmaking based on various morphological characteristics, e.g., flower diameter, colour, florescence and flower type

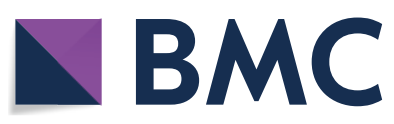

(c) The Author(s) 2019. This article is distributed under the terms of the Creative Commons Attribution 4.0 International License (http://creativecommons.org/licenses/by/4.0/), which permits unrestricted use, distribution, and reproduction in any medium, provided you give appropriate credit to the original author(s) and the source, provide a link to the Creative Commons license, and indicate if changes were made. The Creative Commons Public Domain Dedication waiver (http://creativecommons.org/ publicdomain/zero/1.0/) applies to the data made available in this article, unless otherwise stated. 
a

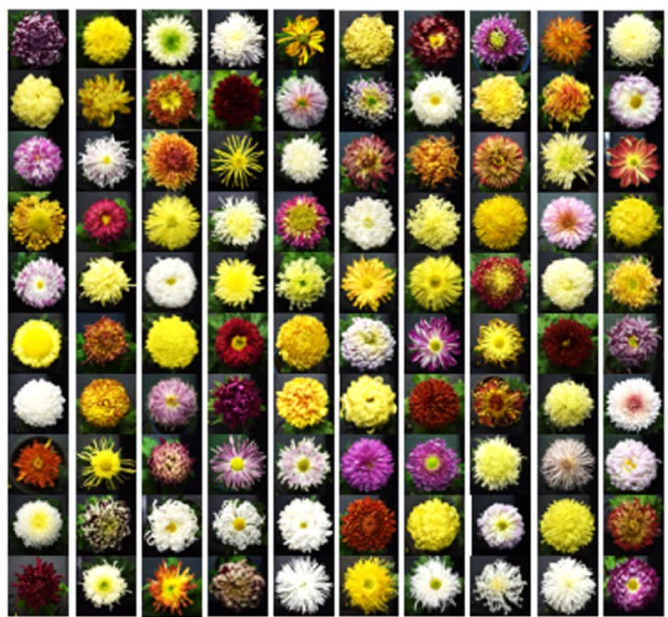

b

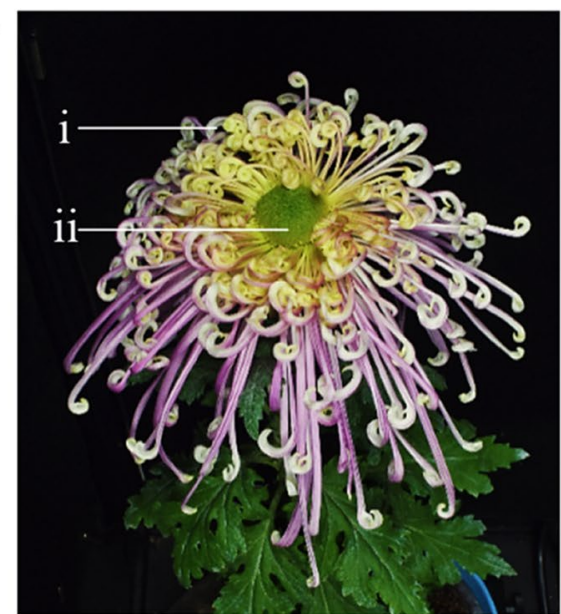

Fig. 1 Complexity of chrysanthemum recognition. a Thousands of large-flowered chrysanthemum cultivars. b The chrysanthemum capitulum structure: (i) ray floret and (ii) disc floret

[11-13]. Numerical taxonomy and multivariate statistical analysis made the recognition process more objective and quantitative [3, 14-16], but required labourious and time-consuming manual measurements. Molecular markers have the potential to recognize cultivars with similar morphological features [17], but required laboratory testing over long periods of at least half a day. At present, the rapid recognition of large-flowered chrysanthemums is difficult to achieve.

Image-based deep learning methods have been increasingly applied in the plant recognition field [18, 19] with the high-speed development of machine learning, which used machine self-learning from massive image data to identify the key features [20]. Compared with previous manual measurement methods, image capture could quickly transform plant morphological information to two-dimensional image information, thus it substantially simplified the process of plant phenotypic data collection [21]. Deep convolutional neural network (DCNN) has been used to identify thousands of plant species. Two deep learning architectures, namely, GoogLeNet and AlexNet, and 8189 images were used to recognize 102 flower species [22]. Flower recognition applications, such as Flowers Partner [23], Flower Recognition [24] and XingSe [25], could recognize more than 4000 plant species based on the deep learning framework. Increases in data availability, along with advances in DCNNs, have made the related approaches more accurate, faster, and cheaper; hence, these approaches have the potential to significantly contribute to solving the problem of flower recognition. Well-trained automated plant recognition systems are now considered to be comparable to human experts in labelling plant on different images [26].
In this paper, we trained a DCNN classifier and used it to recognize large-flowered chrysanthemum cultivars. To the best of our knowledge, the perspective affects the recognition results. A balanced dataset, namely, dataset A, was constructed from 14,000 images of 103 cultivars. The images in dataset A were captured by using an automatic image acquisition device to photograph from predefined perspectives. Each image was reviewed via manual examination to ensure the accuracy of cultivar recognition. Dataset B was constructed from 197 images that were captured in previous years and was used to evaluate the model generalization performance, and the feature distribution of cultivars was observed via T-distributed stochastic neighbour embedding (T-SNE). In addition, the gradient-weighted class activation mapping (Grad-CAM) method and feature clustering were used to interpret the deep learning model's decision-making process from the human perspective. In the study, the main objective was to establish an image-based DCNN classifier for chrysanthemum recognition that would provide high reference value for flower cultivar recognition and plant classification research.

\section{Methods}

Image dataset $\mathbf{A}$

\section{Cultivar selection and plantation}

The experimental material is traditional Chinese largeflowered chrysanthemum cultivar group. 103 cultivars were selected (see Additional file 1: Fig. S1), and some of them were similar in terms of morphology (Fig. 2). The cultivar naming standard of the Chinese Chrysanthemum Book was utilized [5]. 

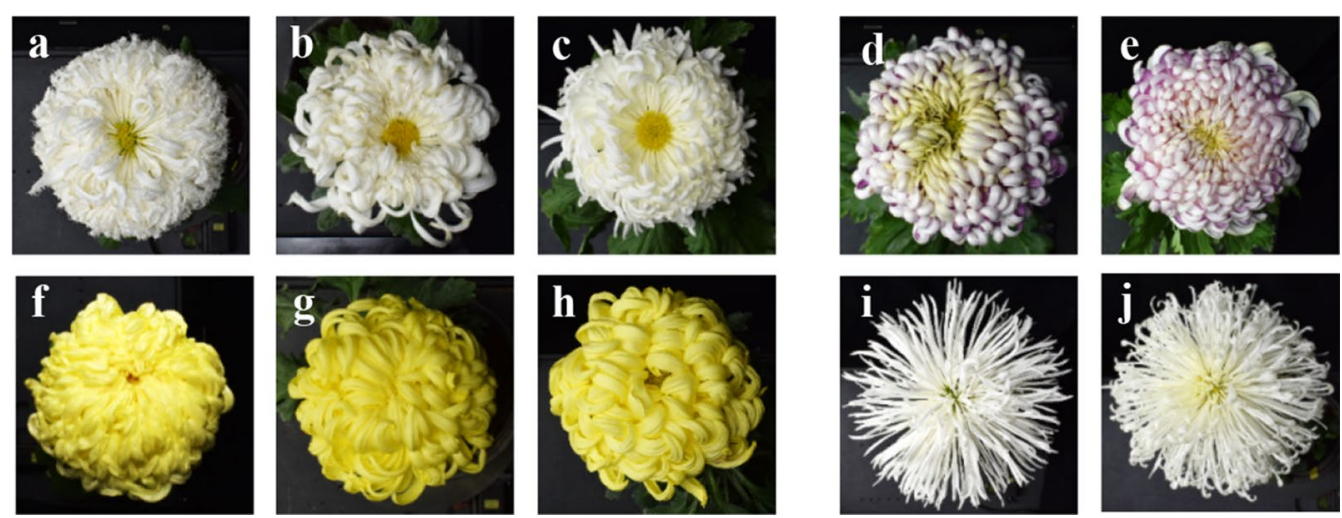

Fig. 2 Similar cultivars in terms of morphology. $\mathbf{a}-\mathbf{c}, \mathbf{b}-\mathbf{d}, \mathbf{e}-\mathbf{g}$, and $\mathbf{h}-\mathbf{j}$ are similar cultivars. The cultivar names are $\mathbf{a}$ 'Baifenshizi', $\mathbf{b}$ 'Qiongdaoshanyou', c'Zilangfengguang', d 'Zilongwoxue', e'Tangyuqiushi', f'Jinfomian', g'Jinshitou', h 'Tangyujinqiu', i'Yulingguan', and j'Baisongzhen'

Plantation work was carried out in the nursery of Beijing Forestry University (Beijing, China) from April 2017 to September 2017. Our cultivation was single-flower cultivation with 10 samples per cultivar. The process included cutting, pot changing, and colonization [8]. Water, fertilizer, insects, and disease control measures were supplied during this period.

\section{Image acquisition}

An automatic image acquisition device, which was designed by our researchers and the GreenPheno Company (Wuhan, China), was used to acquire images (80200 images per cultivar) from October 2017 to December 2017. The flowerpot was placed on a horizontal rotation platform (Additional file 1: Fig. S2), and the rotation angle could be controlled by a computer. When the device rotation stopped, the flowerpot was automatically captured by three cameras from the top, oblique and side views. Because the cultivar height ranged from 0.5 to $1.5 \mathrm{~m}$, we designed a special mode to ensure that the image was focused. First, the flower height was determined by the side-view camera. Then, top-view and oblique-view cameras automatically moved up or down to maintain a specified focusing distance from the top of the flower. Additional details about this device are provided in Additional file 1: Figs. S2 and S3.

After image acquisition, all the images were manually annotated by two researchers over a month, and locally unfocused images were cleaned accordingly.

\section{Dataset construction}

Chrysanthemum dataset A (Fig. 3a) contains 14,000 images (PNG format) of 103 cultivars of Chinese largeflowered chrysanthemum that were captured in 2017. Preserving the percentage of samples for each class, we randomly divided all images into subsets (training, validation and testing).

\section{Image dataset B}

Chrysanthemum dataset B contains 197 images (2-3 images per cultivar) of the same cultivars as in dataset A (Fig. 3b). The images were captured by our group with a digital camera (Canon EOS 750D) in 2008-2010 and in 2016. Compared to the cultivars in 2017, the same cultivars in those years had different cultivating conditions and climatic environment, which led to subtle changes in dataset B. In addition, the images that were captured via manual shooting have higher flexibility compared to machine shooting. To measure the model generalization performance, 197 images were imported into the established classifier.

\section{DCNN approach \\ Devices}

The DCNN models were trained on the Ubuntu 16.04 system on an NVIDIA TitanV GPU (Intel Xeon Gold 5120) hardware platform using the Deep Learning GPU Training System (DIGITS) software, which was developed by NVIDIA.

\section{Framework}

Two pre-trained networks, namely, VGG16 and ResNet50 [27, 28], which were the models in the ImageNet Large Scale Visual Recognition Competition (ILSVRC) in 2014 and 2015, were selected for the detection of a suitable model for chrysanthemum recognition.

In contrast to many common classification tasks of classifying images of various species, such as the ImageNet task, our objective was to classify the images of one species into various cultivars. Hence, the model must extract the fine features of the callosity patterns 
a
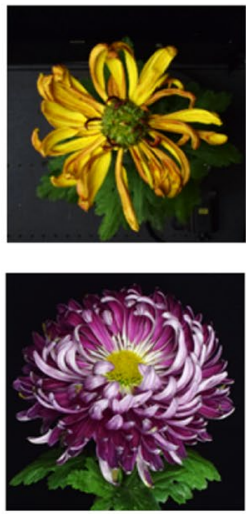

b

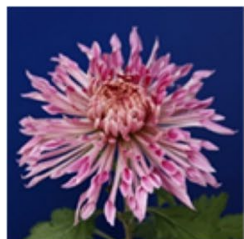

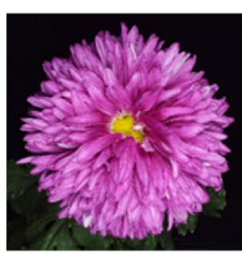
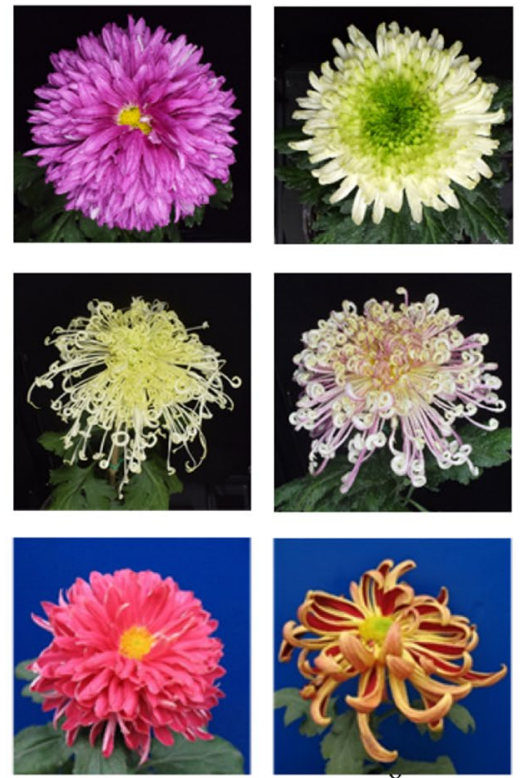
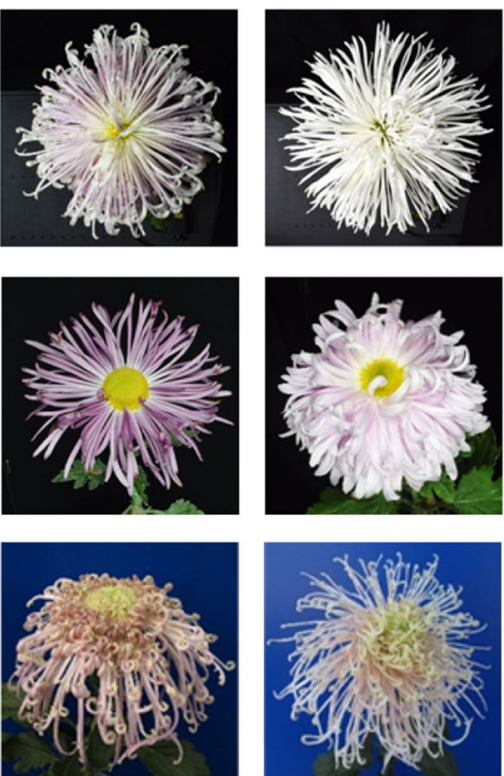

Fig. 3 Sample images from dataset A (a) and B (b)

regardless of the image perspective. Dataset A is a small dataset with only 14,000 images. A common and highly effective approach for deep learning on small image datasets is to use a pre-trained network or transfer learning. A pre-trained network is a saved network that was previously trained on a large dataset, typically on a large-scale image-classification task. The spatial hierarchy of features that was learned by the pre-trained network is used to effectively serve as a generic model of the visual world; hence, its features can be used for many computer vision problems, even completely different classification tasks than the tasks for which it was trained [29]. There are two ways to use the pre-trained network: feature extraction and fine-tuning. Feature extraction was adopted in our DCNN. DCNN is composed of feature extractors and classifiers. The feature extractors that are learned by the pre-trained network have been proven effective in many computer vision problems. Therefore, a classification model can be built on the basis of the pre-trained network. In Fig. 4, VGG16 or ResNet50 acts as a feature extractor. The classifier is comprised of two fully connected layers (each includes 4096 hide units), a global averaging pooling layer and a dropout layer $(0.25)$. The activation function is ReLU.

We used VGG16 and ResNet50 as the base architectures of the network. Although VGG16 is an older model that is far from the current state of the art and is heavier than many recent models, its architecture is simple, and it is easy to understand how the network obtains its final classification decision for a specified image. ResNet50 is famous for obtaining remarkable results on various image classification and object detection tasks. In some respects, ResNet50 is a state-of-theart method.

\section{Training}

DCNN requires a constant input dimensionality. Hence, the flower objects were cropped out from the original images $(6000 \times 4000$ pixel $)$, and the processed images $(2000 \times 2000$ pixel $)$ were down-sampled to a fixed resolution. We trained our DCNN models on the raw RGB values of the pixels. To obtain fixed-size $(224 \times 224$ pixel $)$ DCNN input images, the rescaledsize $(256 \times 256$ pixel $)$ training images were randomly cropped (one crop per image per iteration). To further augment the training set, the crops underwent random transformations, including rotation, translation, shearing, and random RGB colour shifting.

Via the transfer learning approach, a classification model could be built on the basis of the pre-trained network. Two pre-trained networks (VGG16 and ResNet50) were used to compare the classification results. Training used a batch size of 64 with a learning rate of 0.01 and was terminated after 20 epochs. The learning rate adjustment method was the STEP method. As an optimizer for our training algorithms, stochastic gradient descent (SGD) was used. After the training set image was completed, it was verified. The set was tested once in batches. 

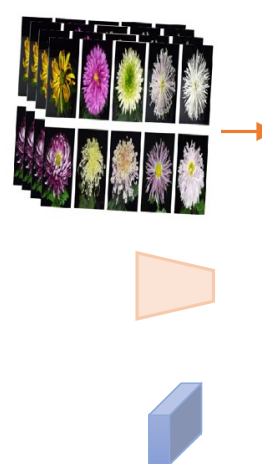

$3 \times 3$ Convolutional

ReLU

Dropout

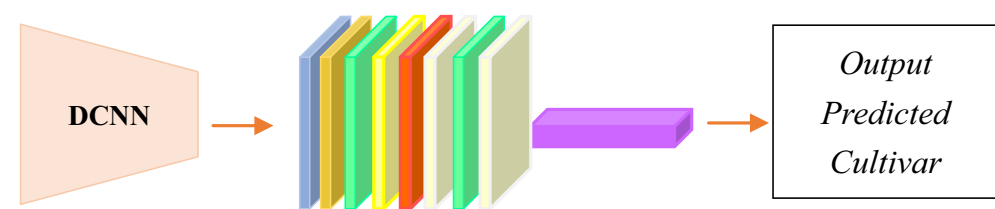

Batch Normalization

Global averaging pooling

Full connected + ReLU

Fig. 4 DCNN framework. Batch normalization was used to improve the performance and stability of DCNN. Dropout prevented DCNN from overfitting. Global average pooling can adapt to any input image size

\section{Evaluation}

As our dataset was completely balanced, we could simply calculate the Top- 1 and Top-5 accuracies for each cultivar as the averages across all images of the test set. Top accuracies have been widely used to evaluate DCNN models in computer vision and image classification; e.g., nearly all papers that present DCNN models that were evaluated on the ImageNet dataset presented their results in terms of both the Top- 1 and Top- 5 accuracies. Therefore, we applied the Top accuracies to our DCNN classifier that was trained on the Chrysanthemum dataset. The Top-1 accuracy is the percentage of predictions for which the top prediction matches the ground-truth label; to calculate this value, the total number of correct predictions is divided by the number of data points in the dataset. When working with Chrysanthemum dataset, which included many class labels with similar characteristics, we could examine the Top-5 accuracy as an extension of the Top-1 accuracy to evaluate the performance of our network (Fig. 5).

\section{T-SNE}

T-SNE could be used to observe the distribution of the chrysanthemum dataset. T-SNE is a method for representing the spatial distribution of features [30], and it projects high-dimensional data into two-dimensional or

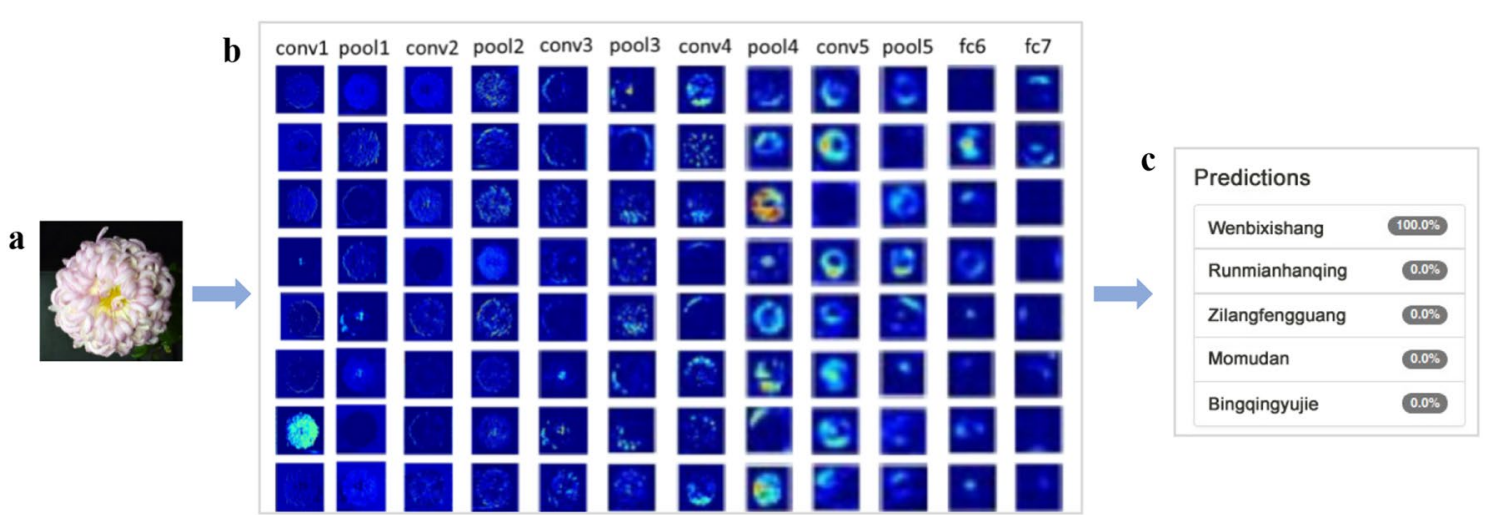

Fig. 5 VGG16 model recognition. a An input image. b The feature extraction visualization results of each convolutional (conv), pooling and fully connected (fc) layer in VGG16 after transfer learning. c The Top-k results 
three-dimensional visualizations to observe the depth features of the cultivars in a spatial distribution, which is used to visually assess the model classification performance. The main strategy is to use the joint probability distribution $p_{i j}$ with symmetry to represent the distances between the sample points in the high-dimensional space [30]. In this paper, we used the T-SNE algorithm to observe the distribution of the high-dimensional features of each image. We extracted the 4096-dimensional features from the 7th layer (fc7) of the chrysanthemum recognition model (VGG16 as a pre-trained network) for each image in the dataset training set (11,200 images), and we visualized the high-dimensional features in twodimensional space. The perplexity was 50 and $n \_$iter was 2000 .

\section{Grad-CAM visualization}

DCNNs are often described as 'black-boxes'; they learn feature representations that are difficult to interpret in a human-readable form. However, the behaviours of DCNNs may be interpreted via Grad-CAM visualization [31]. This approach has been applied to image analysis of soybean plant disease leaves [32]. The main strategy of this approach was to extract the information of the last feature map of the convolutional network (VGG16 as a pre-trained network) to weight the corresponding gradient to produce a location map for displaying key recognition areas in the image [31]. Via Grad-CAM visualization, we could determine which parts of a chrysanthemum image were important when it was identified as belonging to a specified class.

\section{Feature clustering analysis}

A 4096-dimensional feature was extracted in the 7th activation layer of the model (VGG16 as a pre-trained network) from each of the 103 cultivar images ( 5 images per cultivar), and the features of 5 images were averaged for each cultivar. The features could reflect the understanding of model regarding each cultivar image. Hierarchical clustering analysis was conducted on the features of 103 cultivar images using MATLAB 2014a (MathWorks, MA, USA). The calculated distance was set as the cosine distance, the measurement method was ward and the clustering method was the shortest-distance method.

\section{Results}

\section{Model accuracy performance}

Top-k accuracy rate indicators on dataset A were used to the model evaluation. Table 1 lists the calibration accuracies of the VGG16 and ResNet50 networks. The results demonstrate that the recognition accuracies are sufficiently high.
Table 1 Top-1 and Top-5 calibration accuracies for VGG16 and ResNet50 on dataset $A$

\begin{tabular}{lll}
\hline Pre-trained network & Top-1 (\%) & Top-5 (\%) \\
\hline VGG16 & 89.43 & 98.59 \\
ResNet50 & 95.39 & 99.51 \\
\hline
\end{tabular}

Table 2 Top-1 and Top-5 accuracies for VGG16 and ResNet50 on dataset $B$

\begin{tabular}{llll}
\hline Pre-trained network & Top-1 (\%) & Top-5 (\%) & $\begin{array}{l}\text { Analysis time } \\
\text { per image } \\
\text { (ms) }\end{array}$ \\
\hline VGG16 & 51.93 & 78.21 & 10 \\
ResNet50 & 69.86 & 88.19 & 15 \\
\hline
\end{tabular}

\section{Model generalization performance}

The generalization performance is the recognition performance of the DCNN model on new images. Dataset B, which differs substantially from dataset A, was used to measure the model generalization performance. According to Table 2, the two network structures achieved high recognition accuracies. ResNet50 achieved an accuracy of $69.86 \%$. Figure 6 presented the cases that correspond to the Top-5 recognition results. Many cultivars in the Top-5 prediction list were highly similar morphology, especially in terms of flower colour.

\section{Feature distribution}

The distribution of the features were extracted from the various cultivar images by the model that was based on the ResNet50 network. The features of the images of the same cultivar had strong aggregation characteristics (Fig. 7); hence, the features could accurately describe the various chrysanthemum cultivars.

\section{Model decision-making process analysis}

According to the heat maps that were generated by the Grad-CAM method (Fig. 8), the model paid substantial attention to inflorescence edge areas and disc floret areas, and it paid little attention to the leaves and the black background. For the cultivars whose centre disc florets were visible (Fig. 8a), the model focused on the centre disc floret areas, followed by the inflorescence edge areas. For the cultivars whose centre disc florets were invisible (Fig. 8b), the model focused on the inflorescence edge areas. In summary, the inflorescence edge areas and disc floret areas were the key recognition positions.

Clustering analysis was carried out on the 103 cultivar image features, which are presented in a tree diagram (Fig. 9). When the distance was 1.8 to 2.2, 103 cultivar 

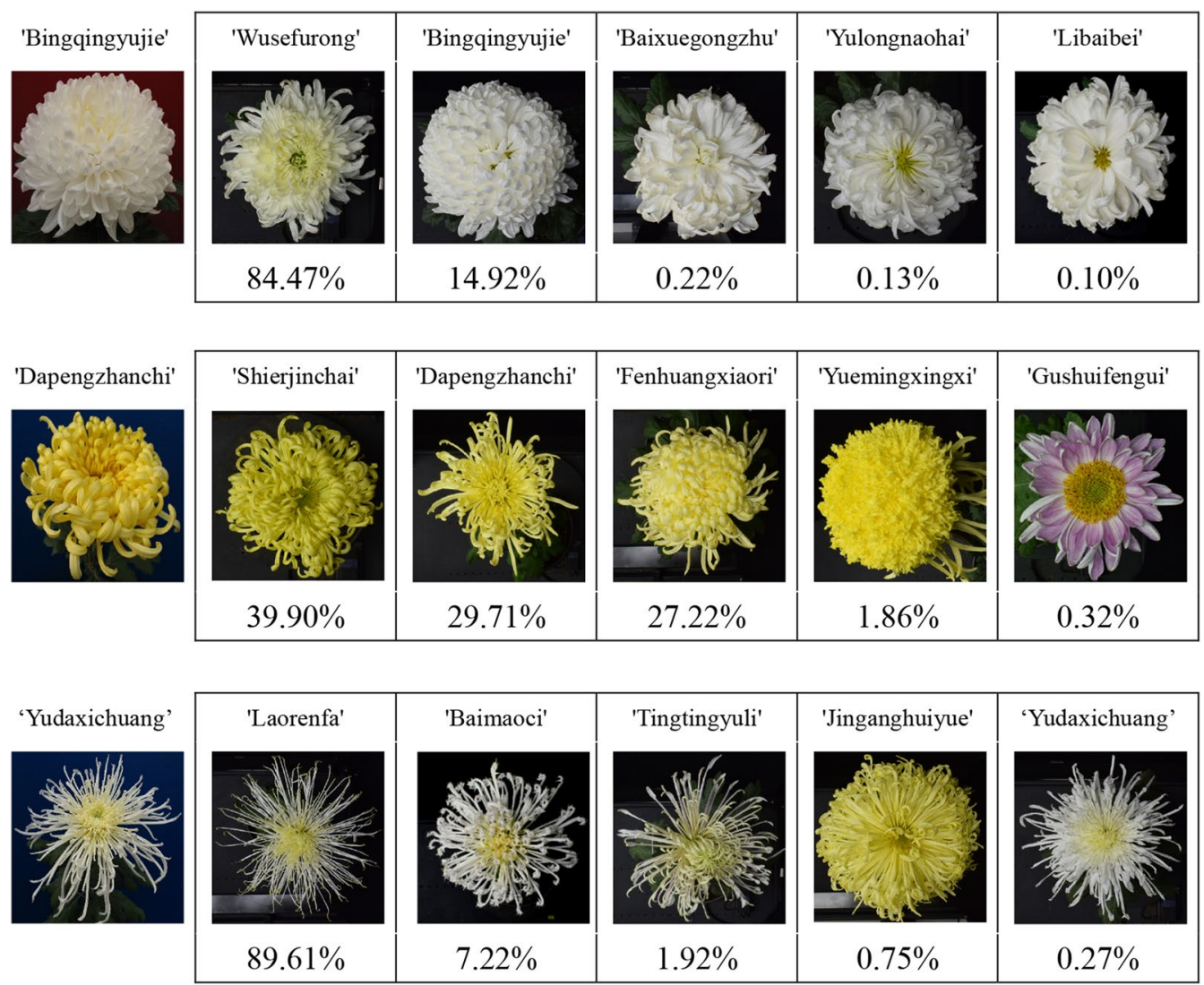

Fig. 6 Top-5 recognition results. On the top of each image, the cultivar name is specified, such as 'Bingjingyujie'; on the bottom, the recognition accuracy is specified

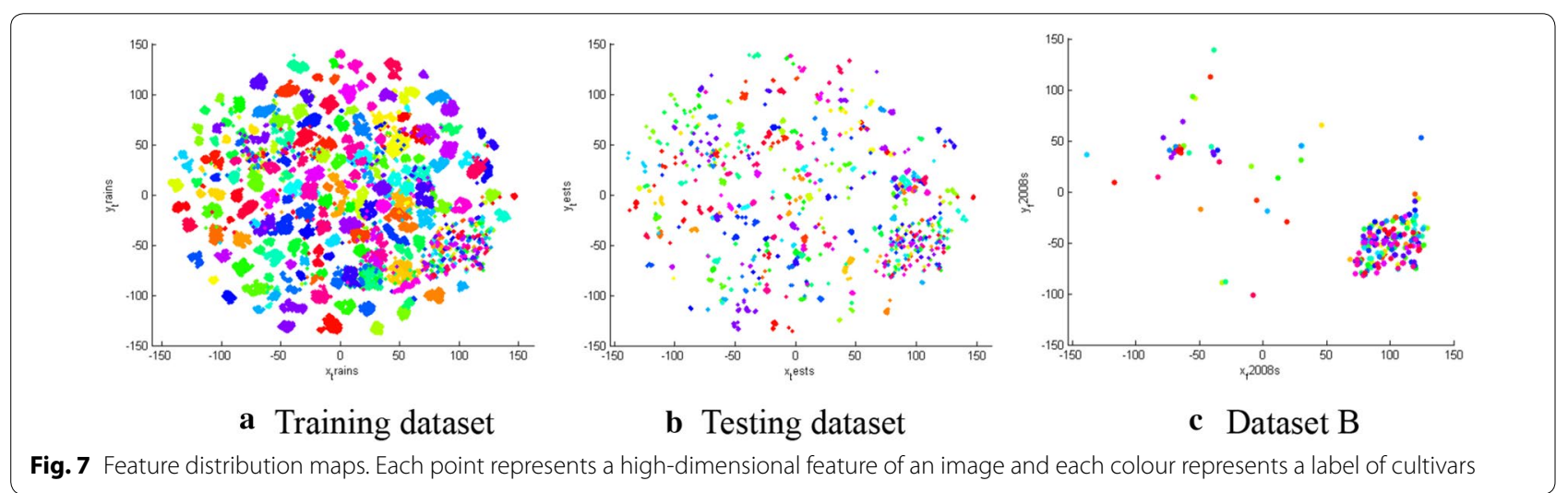

image features were clustered into two categories, which had little readily observable morphology regularity between the corresponding cultivar images (Fig. 9a). When the distance was 1 to 1.2 , the cultivars with similar colour were clustered together, especially white and yellow cultivars (Fig. 9a). When the distance was 0.6 to 0.8 , the cultivars with similar flower shape were clustered together (Fig. 9b-d). In summary, the inflorescence colour and shape were highly correlated with the features that were extracted by the model. 


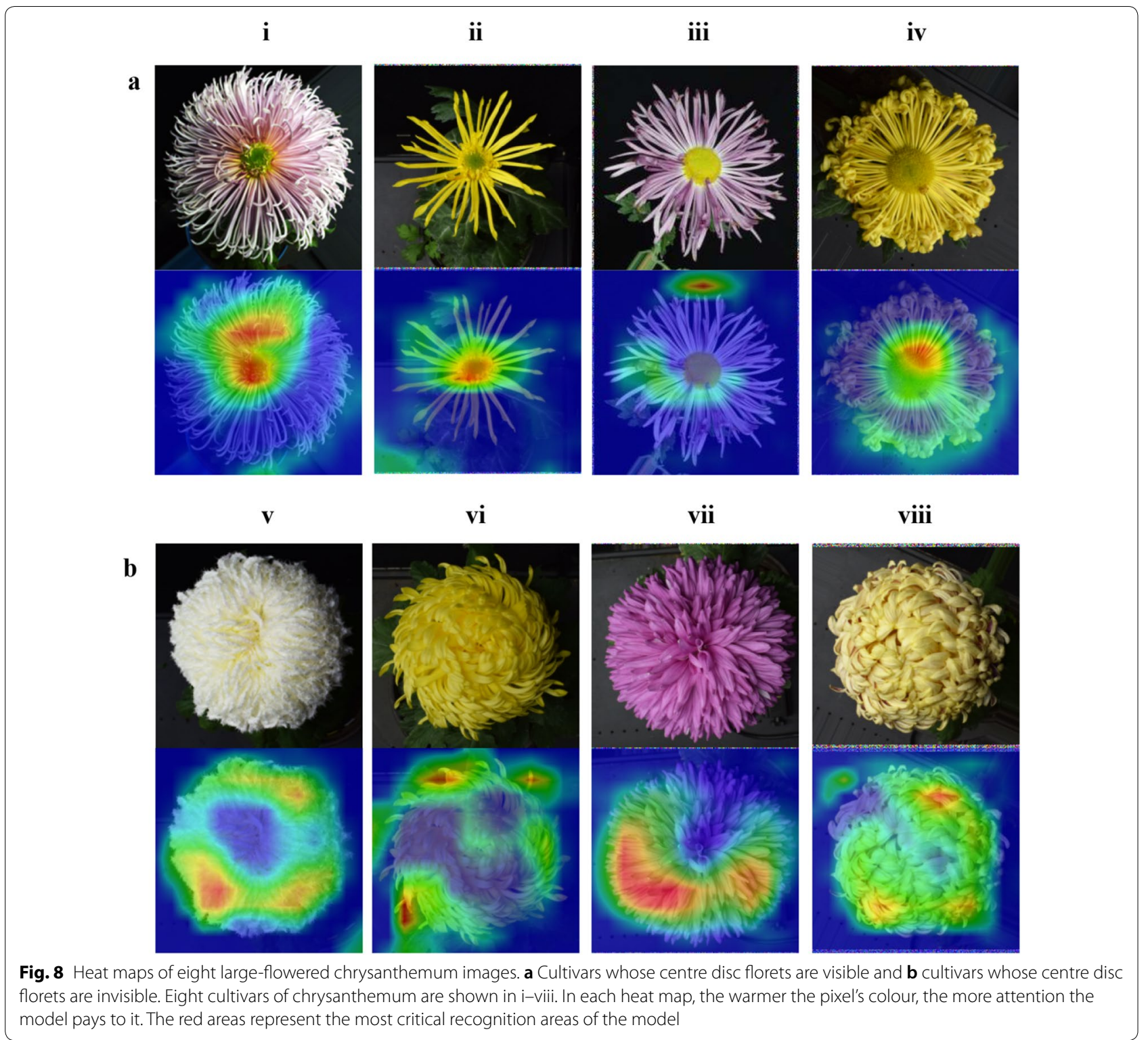

\section{Discussion}

\section{Advantages of image-based deep learning method}

Plant morphological identification is conducted by the naked eye via qualitative comparison to identify the differences in shape among species. It is difficult to transform species information into data for statistical mathematical analysis [33]. Compared with traditional morphological data collection methods, the image acquisition method has the advantages of high speed, high efficiency and ability to carry large amounts of information, thereby substantially simplifying the process plant phenotypic data collection [21, 34].

Deep learning method is a potential research method for identifying plants [35-38]. Compared to inter-species recognition [39], cultivar recognition with high intraclass variability and small inter-class differences is a more challenging task. Compared to traditional methods, which take at least half a day, the DCNN models took $10 \mathrm{~ms}$ (VGG16) and $15 \mathrm{~ms}$ (ResNet50) to analyse a single chrysanthemum image (Table 2), rapidly. In addition, the DCNN that is utilized in this paper can recognize cultivars with high similarity in terms of morphology, whereas applied shallow learning methods $[40,41]$ performed poorly on similar chrysanthemum cultivars. In addition to birds [42], cats and dogs [43] and other classification tasks, this paper demonstrates that the deep learning method also performs well in the classification of chrysanthemum cultivars. 


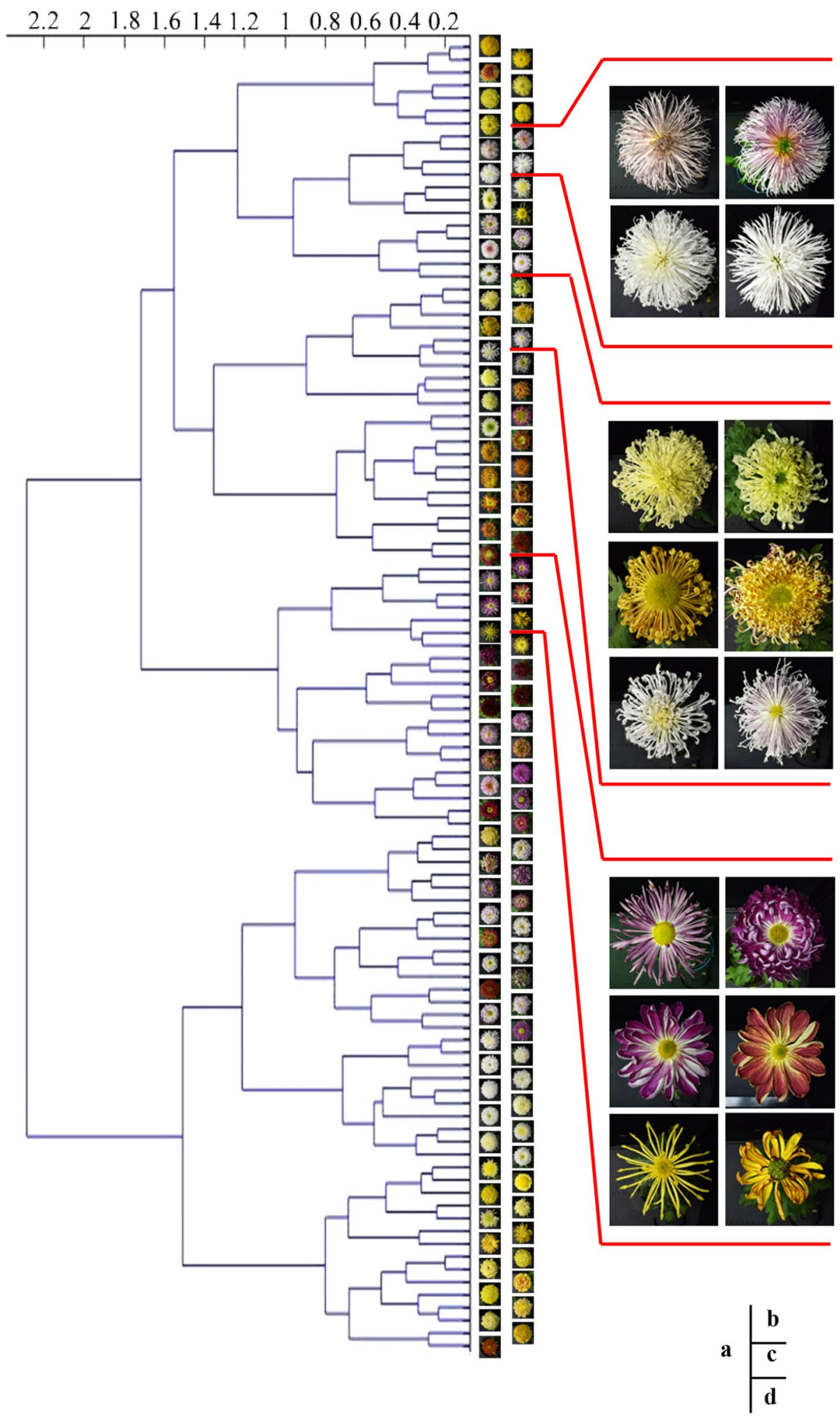

Fig. 9 Clustering analysis of features of 103 cultivar images 


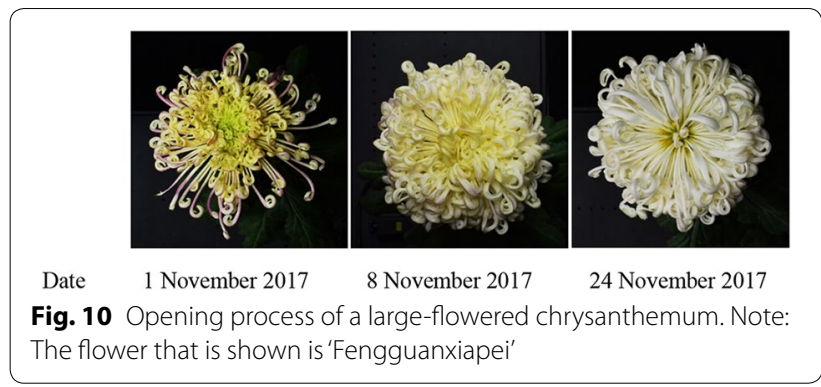

\section{New challenges of chrysanthemum recognition}

Although deep neural networks have high recognition accuracy, their lack of decomposability into intuitive and understandable components renders them difficult to interpret [44]. As the large-flowered chrysanthemum phenotype is highly complex, traditional morphological methods must be combined with multiple traits $[3,14,16]$. How does the deep learning model recognize chrysanthemum? We found that the inflorescence centre and edge areas are important recognition areas and that chrysanthemum images with similar colour or flower shape are always clustered in the same class. This paper reports our initial attempt at clustering analysis. Although our paper did not define the key recognition features of large-flowered chrysanthemums, it demonstrated a fruitful exploration process.

Since hardly any professional chrysanthemum dataset was available online, we established a high-definition large-flowered chrysanthemum image database by recording, storing and sorting chrysanthemum images, which has important significance and value for chrysanthemum image research. A large set of image data is the training basis of a deep learning model. Moreover, the plant phenotype may change with the spatial and temporal conditions. By continuously photographing the same chrysanthemum, we found that some cultivars' phenotypes dramatically changed during their flower opening process, as shown in Fig. 10. Therefore, researchers must collect phenotypic images of the same cultivar in various states, which will pose new challenges in the chrysanthemum recognition task in the future.

\section{Conclusions}

A new method for large-flowered chrysanthemum cultivar recognition is proposed in this paper. The ideal application is that by uploading a single chrysanthemum image to our system, researchers can quickly obtain the Top-5 cultivar information for predicting the cultivar name with the corresponding cultivar images from the system. In addition, newly uploaded images could be reused as input samples for the next iteration, which continuously improves the generalization performance of the model.

\section{Supplementary information}

Supplementary information accompanies this paper at https://doi. org/10.1186/s13007-019-0532-7.

Additional file 1: Fig. S1.Device images of 103 chrysanthemum cultivars (top-view image). Fig. S2. Schematic diagram of the automatic chrysanthemum image acquisition device. Fig. S3. Automatic chrysanthemum image acquisition device.

\section{Abbreviations}

DCNN: deep convolutional neural network; ILSVRC: imageNet Large-Scale Visual Recognition Competition; SGD: stochastic gradient descent; T-SNE: t-distributed stochastic neighbour embedding; Grad-CAM: gradient-weighted class activation mapping.

\section{Acknowledgements}

We thank Greenpheno Company for excellent technical support. We thank Yushan Ji and Hao Liu for helping us plant material. We are particularly indebted to Beijing Dadongliu Nursery for providing test sites. This work was supported by the National Natural Science Foundation of China (No. 31530064) and the Fundamental Research Funds for the Central Universities (No. 2017CGP012)

\section{Authors' contributions}

SD was responsible for the research ideas and the provision of plant germplasm resources. ZL and JW undertook image data collection and image preprocessing. YT and ZL were responsible for the data analysis. ZL completed the first draft. YT, JW and SD revised the manuscript. All authors read and approved the final manuscript.

\section{Availability of data and materials}

The datasets that were used and analysed during the current study are available from the corresponding author upon reasonable request.

Ethics approval and consent to participate

Not applicable.

\section{Consent for publication}

Not applicable.

\section{Competing interests}

The authors declare that they have no competing interests.

Received: 19 June 2019 Accepted: 23 November 2019

Published online: 04 December 2019

\section{References}

1. Boase MR, Miller R, Deroles SC. Chrysanthemum systematics, genetics, and breeding. Plant Breed Rev. 1997;14:321-61.

2. Anderson NO. Chrysanthemum: Dendranthema $\times$ grandiflora Tzvelv. In: Anderson NO, editor. Flower breeding and genetics issues, challenges and opportunities for the 21st century. New York: Springer-Verlag; 2006. p. 389-437

3. Xuebin S, Kang G, Guangxun F, Xiaogang Z, Zhilan L, Silan D. Quantitative classification of the morphological traits of ray florets in large-flowered Chrysanthemum. HortScience. 2018;53(9):1258-65.

4. Pirui L, Fei Z, Sumei C, Jiafu J, Haibin W, Jiangshuo S, et al. Genetic diversity, population structure and association analysis in cut chrysanthemum (Chrysanthemum morifolium Ramat.). Mol Genet Genomics. 2016;291(3):1117-25.

5. Shulin Z, Silan D. Chinese chrysanthemum book. Beijing: China Forestry Publishing House; 2013 (in Chinese).

6. Ackerson C. The complete book of chrysanthemums. New York, NY: American Garden Guild; 1957. 
7. Dejong J, Drennan DL. Genetic analysis in Chrysanthemum morifolium. ii. Flower doubleness and ray floret corolla splitting. Euphytica. 1984:33:465-70.

8. Shuai $Q$, Liwen Y, Xiaohui W, Hong Y, Xuebin S, Mengmeng Z, et al. Reference gene selection for RT-qPCR analysis of flower development in Chrysanthemum morifolium and Chrysanthemum lavandulifolium. Front Plant Sci. 2016; 7:287.

9. Endo M, Inada I. The appearance of sports and chromosome number variations in the Japanese garden chrysanthemum, Chrysanthemum morifolium Ramat. J Jpn Soc Hortic Sci. 1992;61:389-98.

10. Yuan Z, Mingli Z, Silan D. Analysis of karyotype diversity of 40 Chinese chrysanthemum cultivars. J Syst Evol. 2013;51(3):335-52.

11. Niwa T. Chrysanthemum of Japan. Tokyo: The Sanseido Company; 1936

12. Chrysanthemum Classifications [N/OL]. 2019. https://www.mums.org/ chrysanthemum-classes/. Accessed 30 May 2019.

13. Classification Committee National Chrysanthemum Society, Inc. U.S.A. Handbook on Chrysanthemum Classification. 2013.

14. Yuan Z, Xinyan L, Jun Z, Chen W, Yan H, et al. A classification study for chrysanthemum (Chrysanthemum $\times$ grandiflorum Tzvelv.) cultivars based on multivariate statistical analyses. J Syst Evol. 2015;52(5):612-28.

15. Chunying $L$, Lianying $W$. Study on the quantitative classification of chrysanthemum varieties. J Beijing Fore Univ. 1995;17(2):79-877 (in Chinese with an English abstract).

16. Xinyan $L$, Xuebin $S$, Silan D. Variation and probability grading of quantitative characters of traditional Chrysanthemum cultivars. J Beijing Fore Univ. 2016;38:101-11 (in Chinese with an English abstract)

17. Yun Z, Silan D, Yan H, Xuebin S. Application of genomic SSR locus polymorphisms on the identification and classification of chrysanthemum cultivars in China. PLoS ONE. 2014;9(8):e104856.

18. Yu S, Yuan L, Guan W, Haiyan Z. Deep learning for plant identification in natural environment. Comput Intell Neurosci. 2017;2017:7361042.

19. Pawara P, Okafor E, Surinta O, Schomaker L, Wiering M. Comparing local descriptors and bags of visual words to deep convolutional neural networks for plant recognition. In: Proceedings of the 6th international conference on pattern recognition applications and methods (ICPRAM 2017); 2017. p. 479-86.

20. Hinton GE, Salakhutdinov RR. Reducing the dimensionality of data with neural networks. Science. 2006;313(5786):504-7.

21. Andradesanchez P. Development and evaluation of a field-based highthroughput phenotyping platform. Funct Plant Biol. 2014;41:68-79.

22. Gurnani A, Mavani V. Flower categorization using deep convolutional neural networks. 2017. arXiv: 1708.03763.

23. Flowers Partner [N/OL]. 2015. https://hbl.nongbangzhu.cn/. Accessed 30 Nov 2015.

24. Flower Recognition [N/OL]. 2016. https://www.microsoft.com/en-us/ research/project/flowerreco-cn/. Accessed 28 Sept 2016.

25. XingSe [N/OL]. 2018. https://www.xingseapp.com/. Accessed 27 Oct 2018.

26. Goëau H, Joly A, Bonnet P, Lasseck M, Šulc M, Hang ST. Deep learning for plant identification: how the web can compete with human experts. Biodivers Inf Sci Stand. 2018:2:25637.

27. Simonyan K, Zisserman A. Very deep convolutional networks for largescale image recognition. Computer Science. 2014. arXiv: 1409.1556.

28. He K, Zhang X, Ren S, Jian S, editors. Deep residual learning for image recognition. In: 2016 IEEE conference on computer vision \& pattern recognition (CVPR); 2016. p. 770-8.
29. François C. Deep Learning with Python. New York, NY: Manning Publications; 2017.

30. Laurens VDM, Hinton G. Visualizing Data using t-SNE. JMLR. 2008;9(2605):2579-605

31. Selvaraju RR, Cogswell M, Das A, Vedantam R, Parikh D, Batra D. GradCAM: Visual explanations from deep networks via gradient-based localization. In: 2017 IEEE international conference oncomputer vision (ICCV); 2017. p. 618-26.

32. Ghosal S, Blystone D, Singh AK, Ganapathysubramanian B, Singh A, Sarkar $\mathrm{S}$. An explainable deep machine vision framework for plant stress phenotyping. PNAS. 2018;115(18):4613-8.

33. Ming $B$, Xingke $Y$, Jing $L$, Wencheng W. Geometric Morphometrics, a super scientific computing tool in morphology comparison. Chin Sci Bull. 2014;59(10):887-94 (in Chinese with an English abstract).

34. Youxin Y, Zemao Y, Caijun WU, Shuying F. Advances in plant phenomics research. Acta Agri Univ Jiangxiensis. 2015;37(06):1105-12 (in Chinese with an English abstract).

35. Wäldchen J, Mäder P. Plant species identification using computer vision techniques: A systematic literature review. Arch Comput Methods Eng. 2018:25(2):1-37.

36. Gaston KJ, O'Neill MA. Automated species identification: why not? Philos Trans Biol Sci. 2004;359(1444):655-67.

37. Goëau H, Joly A, Bonnet P, Bakic V, Barthélémy D, Boujemaa N, et al. The imageCLEF plant identification task 2013. In: MAED '13 Proceedings of the 2nd ACM international workshop on Multimedia analysis for ecological data; 2013. p. 23-8.

38. Wäldchen J, Rzanny M, Seeland M, Mäder P. Automated plant species identification-Trends and future directions. PLoS Comput Biol. 2018;14(4):e1005993.

39. Šulc M, Matas J. Fine-grained recognition of plants from images. Plant Methods. 2017:13(1):115.

40. Zhilan L, Kang G, Ye T, Silan D, Xuebin S. Identification of chrysanthemum cultivars based on unfolding image with LBP texture feature. 2017 China horticulture horticulture symposium; 2017; Chengdu, Sichuan, China.s (in Chinese with an English abstract)

41. Guo Z, Zhimin L, Wenchao L, Yong Z, Cheng W. Study on varieties identification of ornamental chrysanthemum based on image processing. J Chinese Agri Mech. 2016;37(2):105-10 (in Chinese with an English abstract).

42. Branson S, Van Horn G, Belongie S, Perona P. Bird species categorization using pose normalized deep convolutional nets. In: Proceedings of the british machine vision conference 2014 (BMVC); 2014. arXiv: 1406.2952.

43. Parkhi OM, Vedaldi A, Zisserman A, Jawahar CV. Cats and dogs. In: 2012 IEEE conference on computer vision and pattern recognition (CVPR); 2012. p. 3498-505.

44. Lipton ZC. The Mythos of Model Interpretability. Commun ACM. 2018;61(10):36-42.

\section{Publisher's Note}

Springer Nature remains neutral with regard to jurisdictional claims in published maps and institutional affiliations.

Ready to submit your research? Choose BMC and benefit from:

- fast, convenient online submission

- thorough peer review by experienced researchers in your field

- rapid publication on acceptance

- support for research data, including large and complex data types

- gold Open Access which fosters wider collaboration and increased citations

- maximum visibility for your research: over 100M website views per year

At BMC, research is always in progress.

Learn more biomedcentral.com/submissions 